\title{
Rotura del tendón de Aquiles: a propósito de un esguince de mala evolución
}

\author{
B. Sanz Pozo, A. M oreno García*, L. Rubio Toledano \\ Especialista de Medicina Familiar y Comunitaria. Centro de Salud Greco. Getafe. \\ Área 10 de Madrid. *Residente de tercer año de Cirugía Ortopédica y Traumato - \\ logía. \\ Departamento de Cirugía Ortopédica y Traumatología \\ del Hospital Universitario de Getafe. Madrid
}

\section{RESUMEN}

La rotura del tendón de Aquiles puede ser una entidad difícil de diagnosticar inicialmente, sobre todo en las formas parciales, si no se tiene un alto grado de sospecha clínica. En ocasiones se confunde con otras patologías, como los esguinces de tobillo, por lo que ante un esguince que no evoluciona satisfactoriamente deberíamos pensar en descartar otras patologías no detectadas en un primer momento. Es importante su diagnóstico y derivación precoz al servicio de Traumatología donde se procederá a un tratamiento conservador o quirúrgico, dependiendo de cada caso, para evitar situaciones de incapacidad crónica.

Palabras clave: Tendón de Aquiles. Rotura. Esguince de tobillo. Atención Primaria.
Rupture of Achilles tendon: a case report on ankle sprain with unsatisfactory progress

\begin{abstract}
The rupture of Achilles tendon can be a complaint difficult to diagnose, especially in the partial rupture, if there is not an increased degreee of clinical awareness. There are occasions on which it is misdiagnosed within another pathologies, such as ankle sprain. It is important its diagnostic and early referral to an orthopedist, which will provide a conservative treatment or surgery, according to each case, in order to avoid chronic disabling conditions.
\end{abstract}

Key words: Achilles tendon.Tendon injury. Ankle injuries. Primary Health Care.

\section{INTRODUCCIÓN}

Una buena historia clínica y una exploración sistemática es esencial en la práctica clínica diaria, siendo especialmente útil en la patología traumática recoger en la historia el mecanismo de producción que nos orientará hacia una patología concreta.

El esguince de tobillo es la patología traumática más frecuente del tobillo, produciéndose generalmente por una inversión forzada de éste ${ }^{1,2}$. Toda lesión de tobillo, sin signos de fractura en la radiografía no debe ser diagnosticado de esguince, sin antes realizar una buena exploración descartando otras patologías como fracturas óseas, roturas musculares, tendinosas o incluso roturas ligamentosas.

El médico de Atención Primaria está en una situación privilegiada para seguir la evolución natural de un esguince. Ante un esguince en el que persiste el dolor más allá de 4 a 6 semanas o en el que paciente, pasado este tiempo, no puede caminar correctamente, deberíamos reinterrogarle y explorarle de nuevo, pensando en una mala adherencia al tratamiento o en otras patologías no diagnosticadas inicialmente ${ }^{1,2}$, como rotura del tendón de Aquiles.

La rotura del tendón de Aquiles es una entidad infrecuente que no se diagnostica inicialmente en 
un $25 \%$ de casos, especialmente en las roturas parciales, por lo que es necesario un alto índice de sospecha para el diagnostico correcto $^{3-5}$.Presentamos un caso de rotura de este tendón diagnosticado en Atención Primaria y una revisión de la literatura sobre esta patología

\section{CASO CLÍNICO}

Mujer de 41 años, sin alergias medicamentosas conocidas, ni hábitos tóxicos y sin antecedentes quirúrgicos ni médicos de interés. Presenta entre sus antecedentes personales una lesión de tobillo hace un mes y medio, con dolor y tumefacción en tobillo izquierdo, que fue interpretada como es guince del ligamento peroneo- astragalino anterior, con radiografía de tobillo anteroposterior y lateral sin patología ósea y tratada con inmovilización con férula posterior durante 10 días y posteriormente con vendaje funcional y progresiva movilización hasta el momento actual. Durante los 10 primeros días estuvo tomando ibuprofeno $600 \mathrm{mg} / 8$ horas, no tomando en la actualidad ninguna medicación. Acude a nuestra consulta por persistencia del dolor en tobillo izquierdo, incapacidad para la correcta deambulación y aparición de un "hueco", según relataba la paciente, en la región posterior de tobillo.

En la exploración física se objetivan los siguientes hallazgos: a) en la inspección no se observan signos inflamatorios a nivel del ligamento peroneo astragalino anterior izquierdo, objetivándose únicamente un defecto del contorno en la región posterior del tobillo izquierdo; b) en la palpación aparece dolor y una interrupción a nivel del tendón de Aquiles (Fig. 1); c) explorando la movilidad se objetiva debilidad de la flexión plantar contra resistencia en el tobillo izquierdo en relación con el contralateral e imposibilidad para ponerse correctamente de puntillas. El resto de la exploración es normal.

Ante estos hallazgos en la exploración física sugerentes de rotura del tendón de Aquiles se reinterroga a la paciente sobre el mecanismo de producción de la lesión de tobillo que presentó hace un mes y medio, refiriendo que apareció tras realizar un salto jugando a baloncesto, es decir una flexión plantar súbita del pie contra resistencia, mecanismo frecuente de rotura de este tendón. Por esto se inmovilizó el tobillo con férula posterior y se derivó a la paciente al Servicio de Traumatología, realizando una ecografía que confirmó el diagnóstico de rotura parcial del tendón de Aquiles, procediendo a los catorce días a su reparación quirúrgica. En la cirugía se encontraron los siguientes hallazgos: rotura del tendón a $4 \mathrm{~cm}$ de su inserción, en la práctica totalidad de su espesor quedando íntegras únicamente algunas fibras. Se procedió a realizar una sutura término-terminal con refuerzo. La pa-
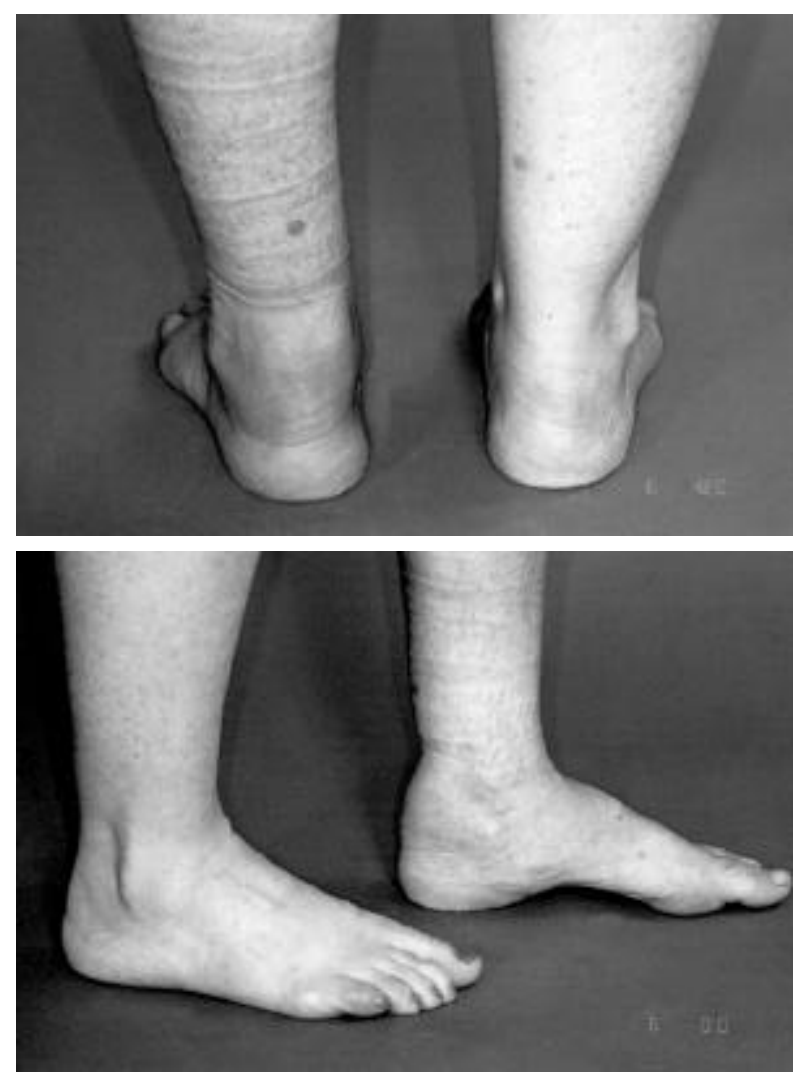

Figura 1

Fotografías de rotura de tendón de Aquiles

ciente evolucionó favorablemente en el postoperatorio y recuperó progresivamente la capacidad de deambulación, tras rehabilitación.

\section{DISCUSIÓN}

Los trastornos musculoesqueléticos son frecuentemente atendidos por los médicos de Atención Primaria ${ }^{6}$.

El tobillo es una de las articulaciones que más traumatismos recibe, produciéndose en la mayoría de los casos distensiones ligamentosas (esguinces) sin llegar a producirse fracturas.

En los esguinces de tobillo se debe recoger en la historia clínica : a) antecedentes de episodios similares (la existencia de episodios previos aumenta el riesgo de aparición); b) localización e intensidad del dolor (la aparición del dolor es inmediato si aparece fractura asociada); c) aparición de tumefacción y/o hematoma, deformidades que sugieran fractura reciente o antigua; d) grado de impotencia funcional (si no es capaz de cargar peso o andar la lesión es de mayor gravedad, como por ejemplo una fractura); y e) el mecanismo de producción. El mecanismo de producción más frecuente es la inversión forzada por lo que el ligamento que con 


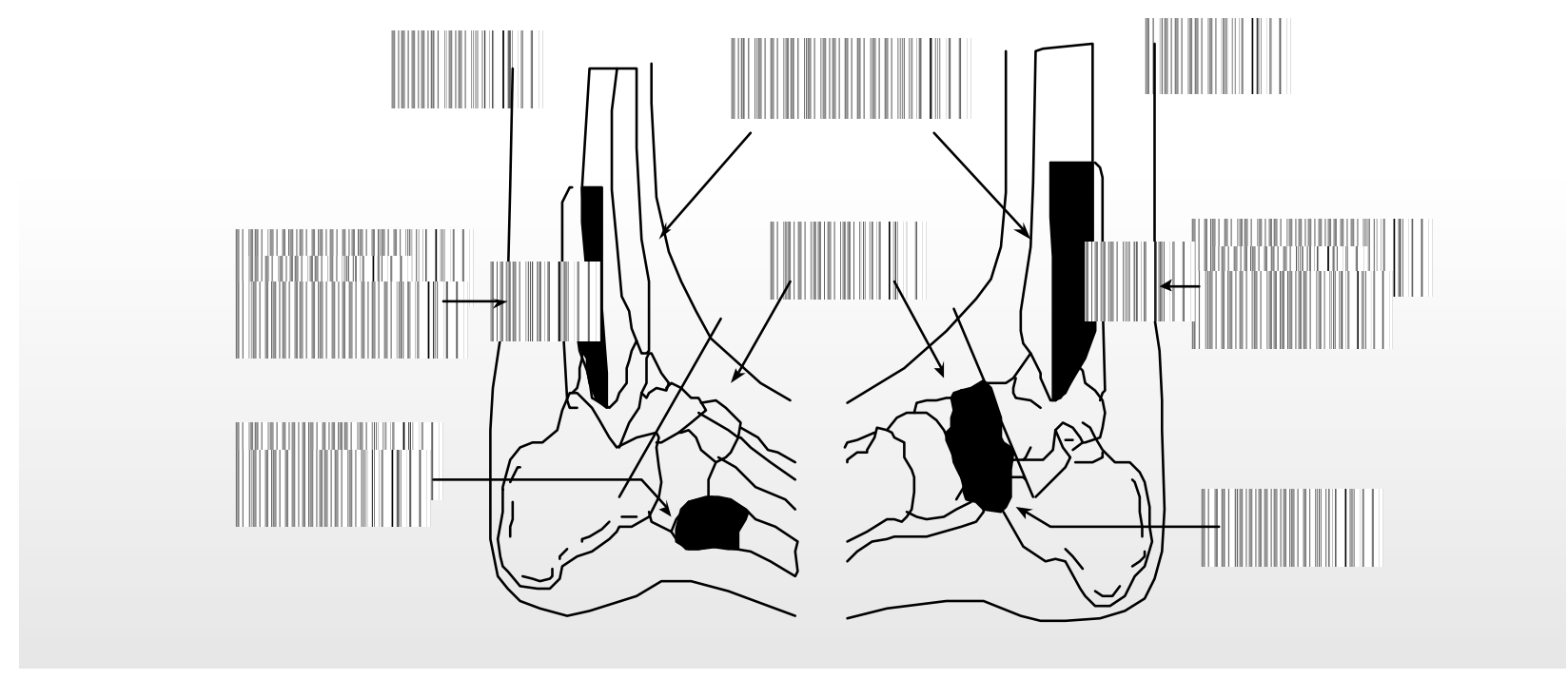

Figura 2

Reglas de Ottawa ${ }^{9}$

más frecuencia se lesiona es el ligamento lateral externo que consta de tres fascículos (según orden de frecuencia lesional; ligamento peroneo-astragalino anterior, peroneo-calcáneo y peroneo-astragalino posterior).

En la exploración del tobillo es importante su realización de forma sistemática con el fin de no llegar a diagnósticos erróneos, por no haber explorado todas las estructuras de cada una de las zonas del tobillo (es útil para la exploración dividir el tobillo en zona lateral, medial y posterior). También es importante comparar con el miembro contralateral y completar la exploración de todas las zonas; habitualmente la zona posterior no suele ex plorarse de rutina si se ha encontrado patología que justifique el dolor en la zona medial y lateral, pudiendo como en el caso clínico que hemos presentado, no diagnosticar de forma precoz una rotura del tendón de Aquiles. Se debe proceder a la palpación de todas las estructuras del tobillo buscando con la punta del dedo la provocación del dolor; a la exploración de las articulaciones (estabilidad y aparición de derrame) y a la exploración de la movilidad pasiva y activa, realizando movimientos para provocar dolor y la localización de éste. Hay que tener en cuenta que la repetición del mecanismo lesional desencadena dolor intenso y que en caso de rotura total el dolor puede ser menor. La movilidad pasiva si es dolorosa y/o está limitada nos indica patología intrarticular; si ésta es normal la excluye.

En Atención Primaria existen unas reglas muy útiles denominadas reglas de Otawa, ${ }^{7-10}$ que son unas guías de práctica clínica para el manejo inicial de los esguinces, utilizadas fundamentalmente para conocer a qué pacientes debemos pedir una radiografía del tobillo o del pie según las características clínicas que poseen. Hay pacientes a los que no se les puede aplicar estas reglas: menores de 18 años, gestantes, heridas aisladas en piel, traumatismo de más de 10 días de evolución, afectación neurológica de los miembros inferiores, difícil valoración del dolor (intoxicaciones, traumatismos craneales, politraumatizados). Son unas reglas simples, fáciles de usar y con una sensibilidad del $100 \%{ }^{7}$. La aplicación de estas reglas ahorra el $30 \%$ de las radiografías, disminuye la incertidumbre del médico, ahorra al paciente el tiempo de espera y evita desplazamientos innecesarios. Dividen el tobillo en dos zonas según lo que se ve en cada radiografía, excluyendo el talón, por lo que hay que tener cuidado con las caídas desde una gran altura con los dos pies, porque puede tener fractura del calcáneo (Fig. 2) $)^{9,10}$ :

1. Zona maleolar $(6 \mathrm{~cm}$ distales tibia y punta del maléolo tibial, $6 \mathrm{~cm}$ distales peroné y punta del maléolo peroneo, astrágalo). Se debe pedir radiografía del tobillo cuando presenta dolor en zona maleolar y una de las tres siguientes: a) incapacidad para cargar el peso del cuerpo y dar 4 pasos inmediatamente después del trauma en la consulta; b) dolor óseo en A (punta maléolo y borde posterior de los $6 \mathrm{~cm}$ distales del peroné); c) dolor óseo en $\mathrm{B}$ (punta maléolo y borde posterior de los $6 \mathrm{~cm}$ distales de la tibia).

2. Zona del mediopie (navicular, cuboides, las tres cuñas, proceso anterior del calcáneo, base del $5^{\circ}$ metatarsiano). Se debe pedir radiografía del pié cuando presenta dolor en zona mediopie y una de las tres siguientes: a) incapacidad para cargar el peso del cuerpo y dar 4 pasos inmediatamente después del trauma en la consulta; b) dolor óseo en $\mathrm{C}$ (base del $5^{\circ}$ metatarsiano); c) dolor óseo en D (navicular).

El tratamiento depende de la gravedad del esguince, para lo cual es útil, aunque difícil, dividir 
Tabla I

HALLAZGOS FíSICOS EN LOS ESGUINCES DE TOBILLO SEGÚN GRADO'

\begin{tabular}{llll}
\hline Signos y síntomas & Gradol & Grado II & Grado III \\
\hline Tendón & No rotura & Rotura parcial & Rotura completa \\
Impotencia funcional & Mínima & Leve & Importante \\
Dolor & Mínimo & Moderado & Severo \\
Inflamación & Mínima & Moderada & Severa \\
Equimosis & Normalmente no & Frecuentemente & Sí \\
Dificultad cargar peso & No & Normalmente & Casi siempre \\
\hline
\end{tabular}

los esguinces en grados (Tabla I ${ }^{1}$ : a) esguinces grado I y II: AINE y hielo local los primeros días, reposo relativo manteniendo el pie elevado el mayor tiempo posible sobre todo durante las primeras 48 horas, con muletas de descarga hasta que desaparezca el dolor y un vendaje inmovilizador e incluso una férula posterior en los de grado II durante 10 días aproximadamente, tras los cuales debe ser reevaluado. Tras este periodo debe ir recuperándose progresivamente, con movilización progresiva realizando ejercicios de fortalecimiento de los múscu$\operatorname{los}^{2}$, e incluso en los de grado I la movilización temprana (en las 24- 48 horas iniciales) puede mejorar el dolor ${ }^{1} \mathrm{y}$ en los de grado II se puede realizar rehabilitación; y b) esguinces grado III: deben ser derivados al traumatólogo, que decidirá según cada caso el tipo de tratamiento a pesar de ser una decisión controvertida, por las diversas posturas encontradas en la literatura revisada, realizando tratamiento conservador con botín de yeso durante 3 a 6 semanas o cirugía (preferiblemente en los pacientes jóvenes, con actividad atlética y cuando está afectado más de un ligamento). Posteriormente se realiza rehabilitación.

La rotura del tendón de Aquiles es más común en los últimos años debido a una combinación de mayor grado de sospecha clínica y una mayor participación en actividades físicas de la población general ${ }^{11}$ Es una de las roturas tendinosas más comunes de la extremidad inferior ${ }^{12}$, describiendo un leve predominio en el miembro inferior izquierdo ${ }^{13}$.

Las roturas parciales del tendón son más difíciles de diagnosticar porque el paciente tiene mínimo dolor y una razonable flexión plantar. Es más frecuente en individuos con tendones previamente debilitados, como personas en edad media con cambios degenerativos o personas que reciben infiltración de corticoides ${ }^{5,13}$. Es también una lesión típica de fin de semana y en individuos con sobrepeso que no realizan un adecuado calentamiento y estiramiento y que realizan deportes de salto y/o carrera.

El diagnóstico se lleva a cabo fundamentalmente con la historia clínica y la exploración, sin necesi - tar en la mayoría de los casos otras pruebas diagnósticas.

En la historia clínica es importante recoger: a) la edad del paciente, ya que como se ha mencionado anteriormente es más frecuente en los individuos de edad media; b) enfermedades o tratamientos previos (se asocia con la toma de fluoroquinolonas); c) intensidad y localización del dolor, que suele ser un dolor brusco a nivel del tendón de Aquiles, que los pacientes describen "como si hubiera recibido un puntapié” en la pierna; y d) percepción de crujidos, que no suelen aparecer porque no suele asociarse con fractura ósea, aunque existen casos en la literatura en los que se asocia con fractura de tobillo; y e) el mecanismo de producción que suele ser la consecuencia de una contracción muscular brusca del músculo tríceps sural (como saltar o correr) al realizar una flexión plantar súbita del pie.

La exploración de la rotura del tendón de Aquiles es mejor realizarla con el paciente en decúbito prono y con los pies colgando por el borde de la camilla, observándose: a) en la inspección el "signo del hachazo", que es un defecto del contorno del tendón y tumefacción; b) en la palpación, se observa en las roturas parciales, en la localización del tendón de Aquiles una zona inflamada dolorosa a la palpación y también se observa una discontinuidad respecto al talón contralateral al realizar flexión plantar contra resistencia, aunque este último signo se aprecia más claramente en las roturas totales ${ }^{16}$; tanto en uno como en otro caso no se aprecia dolor a la palpación de ningún punto óseo; y c) en cuanto a la movilidad presenta una debilidad de la flexión plantar contra resistencia, al indicar al paciente que comprima el pie contra nuestra mano, e imposibilidad para ponerse correctamente de puntillas o no pudiendo andar de puntillas si la rotura es total. La maniobra de Thompson consiste en la aparición de una flexión plantar del pié al apretar la pantorrilla. Esta maniobra distingue entre las roturas completas, en las que esta maniobra es anormal o positiva al no aparecer la flexión plantar, y las roturas parciales en las que es normal, a pesar de tener un defecto palpable $e^{3,16}$. Esta maniobra sería re- 
comendable realizarla en todos los dolores de tobi$1 \mathrm{lo}^{11}$.

En la mayoría de los casos la rotura del tendón de Aquiles puede ser diagnosticada por la clínica y la exploración, pero en las siguientes situaciones el diagnóstico puede ser difícil: hematoma en la zona de la rotura, presencia de cierto grado de flexión plantar realizada por los músculos extrínsecos flexores del pie y maniobra de Thompson falsamente positiva al actuar los músculos flexores accesorios del tobillo (tibial posterior, flexor largo de los dedos y del pulgar).

Las pruebas complementarias que se pueden utilizar para confirmar el diagnóstico son la ecografía y la resonancia magnética (RM). La ecografía es más segura en determinar el espesor del tendón y el tamaño de la solución de continuidad después de una rotura completa. Por el contrario la RM es más fiable en el diagnóstico de las roturas incompletas y la evaluación de los distintos estadios de los cambios degenerativos crónicos. Generalmente el tendón es inicialmente valorado con ecografía de bido a que es una técnica fácil, barata y que permite el examen dinámico, aunque necesita una importante experiencia del ecografista; y si los hallazgos encontrados son dudosos se debería realizar una $\mathrm{RM}^{18}$.

En el tratamiento lo principal es su derivación lo antes posible al servicio de Traumatología, para proceder a un tratamiento de tipo conservador o quirúrgico de forma precoz que evite situaciones de incapacidad crónica ${ }^{3}$. El objetivo del tratamiento es restaurar la longitud, tensión, fuerza y función del tendón ${ }^{16}$, existiendo en la actualidad controversia sobre cuál es el tipo de tratamiento que mejor alcanza estos objetivos. Revisando la literatura existente se observa que el tratamiento quirúrgico ofrece una mejor capacidad funcional, pero ambos tipos de tratamiento no garantizan la ausencia de complicaciones: nueva ruptura, debilidad de la flexión plantar, rigidez del tobillo, mala cicatrización de la herida (a veces tarda meses) y trombosis venosa profunda.

El tratamiento conservador suele aconsejarse en los pacientes ancianos, sedentarios, los de alto riesgo anestésico y en los casos diagnosticados y atendidos en las primeras 48 horas desde la lesión ${ }^{5,11,13}$.
Este tratamiento presenta un riesgo mayor de reruptura y el paciente presenta una disminución de la fuerza del tendón.

El tratamiento quirúrgico se aconseja en los pacientes jóvenes y deportistas ${ }^{11,16}$ y proporciona los resultados más satisfactorios respecto al tratamiento conservador cuando el diagnóstico o el tratamiento se demora una semana o más ${ }^{13}$ como en el caso clínico presentado. Entre sus ventajas respecto al tratamiento conservador se encuentra una menor tasa de re-ruptura ( 0 a $2 \%$ vs 8 a $39 \%$ ), una mayor aunque leve recuperación de la fuerza del tendón y un porcentaje alto de pacientes que pueden volver a practicar deportes ${ }^{11,20}$. Entre sus inconvenientes se encuentra una mayor tasa de complicaciones menores como infecciones de la herida, necrosis de la piel y lesiones nerviosas, aunque con una técnica quirúrgica adecuada estas complicaciones pueden minimizarse $\mathrm{e}^{13,16}$. Existen distintas técnicas quirúrgicas, incluso en los últimos años se está utilizando reparación percutánea. La literatura reciente sugiere que la movilización de forma temprana y gradual tras la cirugía es efectiva y no aumenta el riesgo de re-ruptura. Incluso en los deportistas de élite comienzan a los 3 ó 7 días de la reparación quirúrgica $^{16}$.

El diagnóstico de rotura de tendón de Aquiles, aunque infrecuente, no es difícil siempre que se considere esta posibilidad al valorar una lesión de pie/tobillo, siendo importante su diagnóstico precoz y manejo adecuado.

\section{AGRADECIMIENTOS}

A mi tutor, Jose Luis Quintana Gómez

\section{CORRESPONDENCIA}

Blanca Sanz Pozo

C/ Alicante $15,4^{\circ} \mathrm{B}$

28903 Getafe. Madrid

Tel: 91-695 2787

e-mail: bsanzpozo@hotmail.com 


\section{Bibliografía}

1. Wexler RK. The injuried ankle. Am Fam Physician 1998; 57(3): 474-80.

2. Swain RA, Holt WS. Ankle injuries. Postgrad Med 1993; 93(3): 91-100.

Med 1994; 18: 173-201.

5. Hang DW, Bach BR, Bojchuk J. Partial Achilles tendon rupture following corticosteroid injection. A caveat to practitioners. Phys Sports Med 1995; 23(2): 57-8; 636.

6. Musculoskeletal problems are common in family practice. (Conference Highlights). Am Fam Physician 1996; 54(8): 2524.

7. Decision rules for the use of radiography in acute ankle injuries: refinement and prospective validation. JAMA 1993; 269(9): 1127-32.

8. Stiell IG, Greenberg GH, McKnight RD, Nair RC, McDowell I, Worthington JR. A study to develop clinical decision rules for the use of radiography in acute ankle injuries. Ann Emerg Med April 1992; 21: 384-90.

9. Stiell IG, McKnight RD, Greenberg GH, McDowell I, Nair RC, Wells GA, et al. Implementation of the Ottawa ankle rules. JAMA 1994; 271: 827-32.

10. Stiell IG, Wells GA, Laupacis A, Brinson R, Verbeek R, Vandemheen $\mathrm{K}$, et al. Multicentre trial to introduce the Ottawwa ankle rules for use of radiography in acute ankle injuries. BMJ 1995; 311: 594-7.

11. Popovic N, Lemaire R. Diagnosis and treatment of acute ruptures of the achilles tendon current concepts review. Acta-Orthop-Belg 1999; 65(4): 458-71.

12. Yoho RM, Slate RE. Achilles tendon rupture. Diagnosis and managament. Clin Podiatr Med Surg 1999; 16(4): 617-30.

13. Leppilahti J, Orava S. Total Achilles tendon rupture: a review. Sports Med 1998; 25(2): 79-100.

14. Biundo JJ, Mipro RC, Fahey P. Sports- related and
3. Ballas M, Tytko J, Mannarino F. Commonly missed orthopedic problems. Am Fam Physician 1998; 57(2): 267-74.

4. Kvist M. Achilles tendon injuries in athletes. Sports other soft-tissue injuries, tendinitis, bursitis, and occupation-related syndromes. Curr Opin Rheumatol 1997; 9(2): 151-4.

15. Casparian JM, Luchi M, Moffat RE, Hinthorn D. Quinolones and tendon ruptures. Sputh Med J 2000; 93(5): 488-91.

16. Saltzman CL, Tearse DS. Achiles tendon injuries. J Am Acad Orthop Surg 1998; 6(5): 316-25.

17. Lubin JW, Miller RA, Robinson BJ, Blevins FT. Achilles tendon rupture associated with ankle fracture. Am J Orthop 2000; 29(9): 707-8.

18. Neuhold A, Stiskal M, Kainberger F, Schwaighofer B. Degenerative Achilles tendon disease: assessment by magnetic resonance and ultrasonography. Eur $\mathrm{J}$ Radiol 1992; 14: 213-20.

19. Lo IK, KirKley A, Nonweiler B, Kumbhare DA. Operative versus nonoperative treatmente of acute Achilles tendon ruptures: a quantitative review. Clin J Sports Med 1997; 7(3): 207-11.

20. Cetti R, Christensen SE, Ejsted R, Jensen NM, Jorgensen $U$. Operative versus nonoperative treatment of Achilles tendon rupture: a prospective randomized study and review of the literature. Am J Sports Med 1993; 21: 791-9.

21. Cetti R, Henriksen LO, Jacobsen KS. A new treatment of ruptured Achilles tendons: a prospective randomized study. Clin Orthop 1994; 308: 155-65.

22. Mandelbaum BR, Myerson MS, Forster R. Achilles tendon ruptures: a new method of repair, early range of motion, and functional rehabilitation. Am J Sports Med 1995; 23: 392-5. 